\title{
Acute Mediastinitis Following a Laparotomy for Small Bowel Obstruction
}

\author{
Sohel Samad ${ }^{\mathrm{a}}$, Marianthi Vatrika ${ }^{\mathrm{a}}$, Rizwan Basit ${ }^{\mathrm{a}}$, Sudhakar Mangamª, Samer Doughan ${ }^{\mathrm{a}, \mathrm{b}}$
}

\begin{abstract}
Acute mediastinitis is a rare and severe life-threatening infection affecting the connective tissues that surround the vital structures of the mediastinum. The disease is most commonly a complication of cardiothoracic and esophageal surgery, esophageal perforation or secondary to upper cervical or dental infections and has a mortality rate of $12-40 \%$. Although cases of mediastinitis secondary to cardiothoracic surgery and esophageal perforation secondary to foreign body and endoscopy have been described, in this case we describe an unusual presentation of mediastinitis in a patient who underwent a laparotomy for a small bowel obstruction and developed postoperative acute mediastinitis with a retropharyngeal and paraesophageal abscess. This case highlights the importance of early identification and management of acute mediastinitis and demonstrates that upper gastrointestinal interventions are not without risk.
\end{abstract}

Keywords: Mediastinitis; Small bowel obstruction; Laparotomy; Nasogastric tube; Central line; PICC line; Atrial fibrillation

\section{Introduction}

Acute mediastinitis is a rare and severe life-threatening infection affecting the connective tissues that surround the vital structures of the mediastinum [1,2]. The disease is most commonly a complication of cardiothoracic and esophageal surgery, esophageal perforation or secondary to upper cervical or dental infections [1, 3, 4]. Mediastinitis confers a high mortality rate ranging from $12 \%$ to $40 \%[4,5]$, with mortality rates significantly improved by early diagnosis, aggressive antibiotic treatment and surgical intervention $[1,3,4,6]$. Although cases of mediastinitis secondary to cardiothoracic surgery and

Manuscript accepted for publication November 26, 2014

aEast Kent Hospitals University Foundation Trust, Queen Elizabeth the Queen Mother Hospital, Margate CT9 4AN, UK

${ }^{b}$ Corresponding Author: Samer Doughan, General Surgical Department, Queen Elizabeth the Queen Mother Hospital, Margate CT9 4AN, UK.

Email: s.doughan@btinternet.com

doi: http://dx.doi.org/10.14740/jcs252w esophageal perforation secondary to foreign body and endoscopy have been described, in this case we describe an unusual presentation of mediastinitis in a patient who underwent a laparotomy for a small bowel obstruction and developed postoperative acute mediastinitis with a retropharyngeal and paraesophageal abscess.

\section{Case Report}

A 75-year-old female presented with 10 days history of persistent vomiting, weight loss of 1 stone in 1 week and bowels not opening for 2 days. Her past medical history included hysterectomy with mesh sacro-colpopexy and appendicectomy.

On examination, her abdomen was mildly distended with scanty bowel sounds and a rectal exam was unremarkable. A computerized tomography (CT) scan on admission revealed a small bowel obstruction with dilated small bowel loops. With a provisional diagnosis of adhesive small bowel obstruction, she was taken for an emergency laparotomy. Emergency laparotomy revealed extensive adhesions with small bowel stuck to the mesh from previous pelvic sacro-colpopexy. A limited small bowel resection was carried out with a primary anastomosis.

Postoperatively the patient developed an acute kidney injury and was admitted to the intensive care unit (ICU) where a central venous line (CVP) and a nasogastric (NG) feeding tube under Cortrak $^{\circledR}$ guidance was inserted. Of note is that it required at least four attempts to insert the NG tube. The patient recovered well with ICU support and antibiotics. She was subsequently discharged to the ward where NG feeding was continued (further two replacements of NG tube were needed due to displacement).

However, on postoperative day 9 when oral feeding was attempted following a speech and language therapy assessment, the patient suffered from persistent regurgitation and discomfort within the oral cavity. Clinical examination revealed a likely oral fungal infection and the patient was commenced on nystatin and oral fluconazole. NG feeding was continued for nutrition. Although throat swabs and blood cultures were unremarkable, blood investigations revealed a raised C-reactive protein (CRP) with low albumin.

On postoperative day 12 the patient developed fast atrial fibrillation (AF) with no underlying electrolyte abnormality. There was no evidence of intraperitoneal sepsis or cardiac is- 

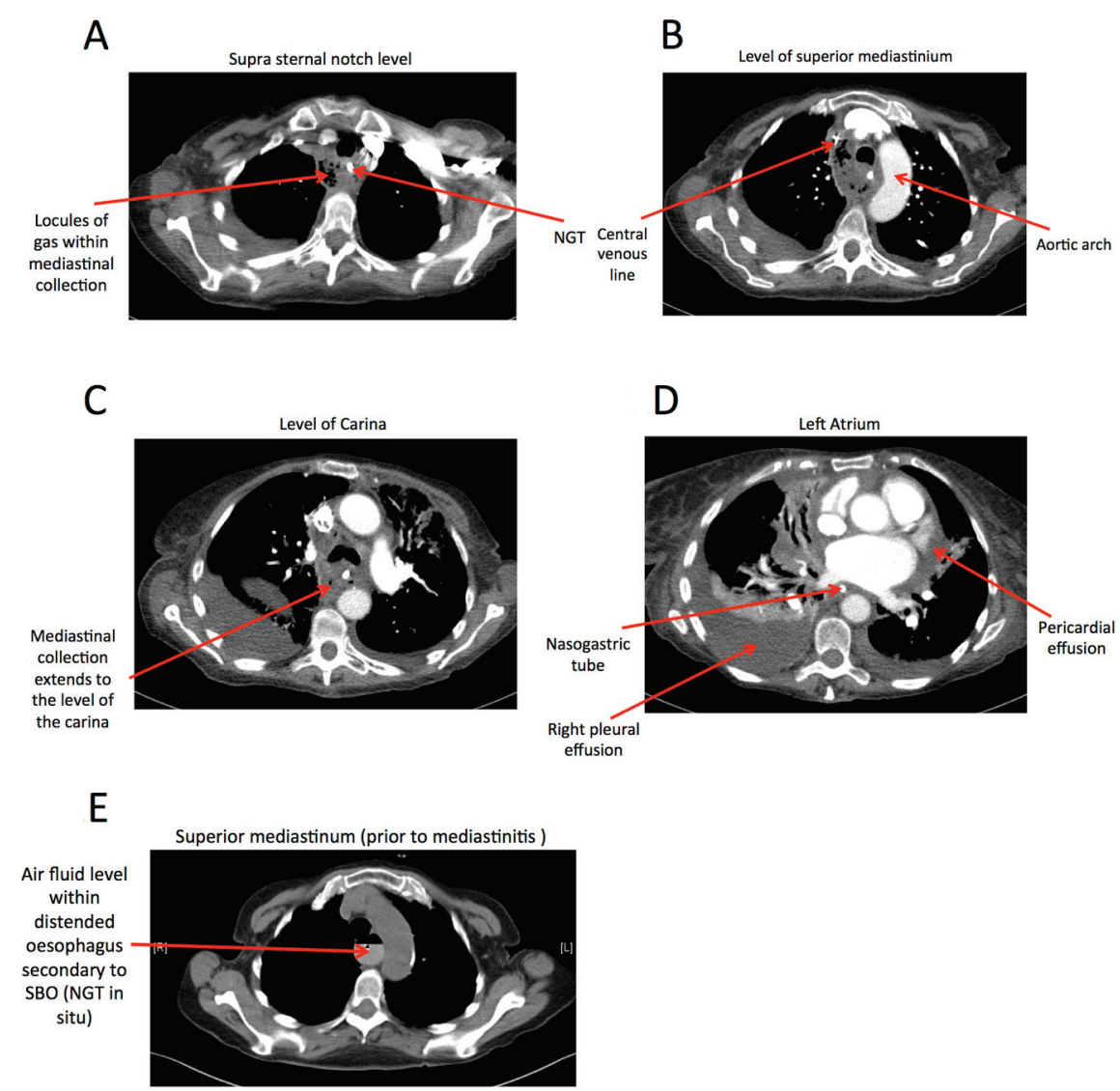

Figure 1. (A) Mediastinal collection at supra-sternal notch level. (B) Mediastinal collection and gas at the superior mediastinum. (C) Mediastinal collection at the level of the carina. (D) Pericardial effusion. (E) Superior mediastinum prior to development of acute mediastinitis demonstrates no collection or gas within the mediastinum.

chemia that could account for the AF. Apart from a raised CRP, all other radiological and biochemical investigations were within normal limits. The patient was treated with bisoprolol and her AF was controlled.

However, despite continued supportive treatment, on postoperative day 14, the patient became tachypnoeic and tachycardic; subsequently, an urgent CT pulmonary angiogram (CTPA) was performed. The CTPA excluded a pulmonary embolism but surprisingly demonstrated a mediastinal collection extending from the root of the neck to the bifurcation of the trachea consistent with mediastinitis (Fig. 1). A retropharynge- al abscess was also demonstrated at the mid cervical level (Fig. 2 ) without any $\mathrm{CT}$ evidence of an esophageal perforation. The patient was immediately started on meropenem and a thoracic opinion was sought. Unfortunately at the time of CT diagnosis, the patient was deemed to be no longer fit for anesthesia or further surgical intervention. She passed away the following day. A post-mortem examination confirmed mediastinitis secondary to a retropharyngeal and esophageal abscess, and no clear esophageal perforation could be seen. However, it was deemed by the pathologist that the retropharyngeal abscess may have been secondary to trauma.

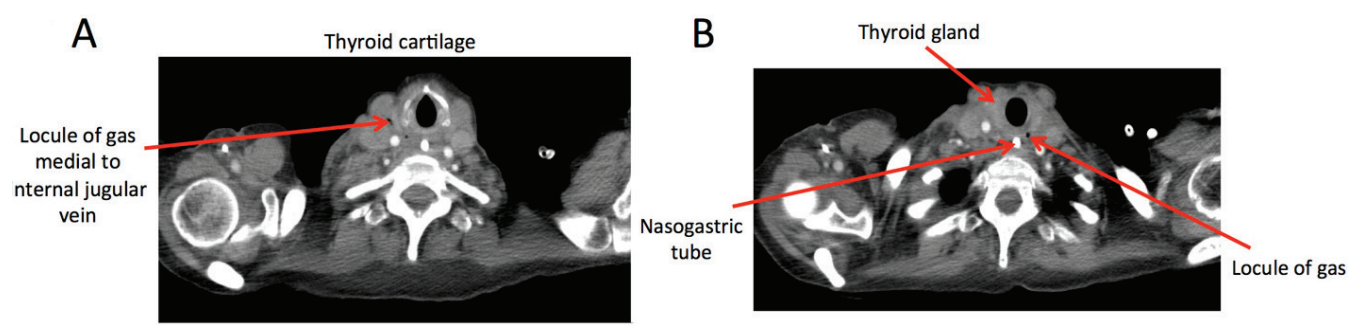

Figure 2. (A) Locules of gas observed within the thyroid cartilage level. (B) Locules of gas observed within the thyroid gland level. 


\section{Discussion}

Acute mediastinitis is a rare but life-threatening illness with a high mortality rate $[1,4,5]$. However early diagnosis and aggressive management with antibiotic therapy and surgical intervention can significantly reduce mortality $[1,3,4,6]$.

One of the key features to treating this disease is early recognition [7]. The classical presentations of acute mediastinitis are fever, tachycardia, sepsis and in some cases retrosternal chest pain [8]. Despite the negative blood and throat cultures, the presence of an unexplained AF as discussed in this case could have been a physiological reaction to a developing mediastinitis. Furthermore, the pain and difficulty in swallowing demonstrated by the patient in this case could have been secondary to a developing retropharyngeal and paraesophageal abscess [4].

In addition to early recognition of clinical signs, the key to diagnosing mediastinitis is radiological imaging, in particular with CT scanning $[5,8,9]$. Akman et al describe the CT findings of mediastinitis to comprise of mediastinal widening, air fluid level, or possibly air within the mediastinum secondary to perforation or bacterial infection [8]. Moreover, a study by Misawa et al on postoperative mediastinitis secondary to cardiac operations concluded that mediastinal swelling detected on CT was consistent with mediastinitis; however, they also concluded that they did not see any correlation between fluid collection in the mediastinum and mediastinitis [9]. CT scanning has a clear role in the diagnosis of mediastinitis. Miller et al [10] reported that the combination of CT and clinical examination had an accuracy rate of $89 \%$ with a sensitivity rate of $95 \%$ in the diagnosis of mediastinitis. Furthermore, CT scanning not only allows the diagnosis of mediastinitis to be confirmed, but can also elude as to the possible origin of the disease as demonstrated in this case by the presence of a widened mediastinum. Air in the mediastinum (pneumomediastinum) and the simultaneous presence of a retropharyngeal abscess as detected on CT may imply that the mediastinitis could have been secondary to a retropharyngeal abscess.

A final point of discussion in this case is the possibility of trauma secondary to insertion of feeding NG tube. If this patient had a small Zenker's diverticulum (pharyngeal pouch), this could have resulted in small traumatic perforation during insertion of NG tube leading to the retropharyngeal abscess and subsequently mediastinitis. However, there was no evidence of a pharyngeal pouch from post-mortem. It is also possible that repeated attempts at insertion of NG tube (as in this case) could have resulted in oesophageal trauma.

Cases of mediastinitis secondary to retropharyngeal abscess [4, 11], esophageal perforation [1], and cardiothoracic surgery $[5,12]$ are documented. Cases of mediastinitis following an emergency laparotomy however have not been reported. Despite the correction of presenting pathology, this patient did not improve. This could have prompted earlier investigations into the cause of ongoing sepsis. Further mediastinitis and a retropharyngeal abscess were simultaneously present in this case, implying that the mediastinitis was possibly secondary to the retropharyngeal and paraesophageal abscess. The patient in this case had an NG feeding tube inserted after four attempts. It is possible that trauma from this NG tube insertion could have led to the retropharyngeal and paraesophageal abscess and the subsequent descending mediastinitis. Furthermore Sposato et al [13] reported a case of mediastinitis secondary to a peripherally inserted central venous catheter (PICC) insertion, the patient in this case also had a PICC line and therefore it is possible that this may have been the source of the mediastinitis. However, the post-mortem examination did not reveal any evidence of trauma secondary to a PICC line insertion. Finally Eroglu et al Eroglu al [14] reported a case of esophageal rupture following persistent vomiting. It is a possibility that the persistent vomiting observed in this case led to an esophageal perforation (Boerhaave's syndrome) and this led to the subsequent mediastinitis.

\section{Conclusion}

Mediastinitis continues to convey a high mortality rate, although improvements in CT diagnosis and management of mediastinitis have aided in reducing this mortality. There needs a high index of suspicion for rare diagnosis like mediastinitis in critically ill postoperative patients especially in the presence of upper gastrointestinal interventions and the absence of a clear underlying pathology. The case we have presented further demonstrates the need to continue our research into the disease and reiterates the importance of early diagnosis and aggressive treatment.

\section{References}

1. Macri P, Jimenez MF, Novoa N, Varela G. [A descriptive analysis of a series of patients diagnosed with acute mediastinitis]. Arch Bronconeumol. 2003;39(9):428-430.

2. Diamantis S, Giannakopoulos H, Chou J, Foote J. Descending necrotizing mediastinitis as a complication of odontogenic infection. International Journal of Surgery Case Reports. 2011;2:65-67.

3. Poluri A, Singh B, Sperling N, Har-El G, Lucente FE. Retropharyngeal abscess secondary to penetrating foreign bodies. J Craniomaxillofac Surg. 2000;28(4):243-246.

4. Estrera AS, Landay MJ, Grisham JM, Sinn DP, Platt MR. Descending necrotizing mediastinitis. Surg Gynecol Obstet. 1983;157(6):545-552.

5. El Oakley RM, Wright JE. Postoperative mediastinitis: classification and management. Ann Thorac Surg. 1996;61(3):1030-1036.

6. Marty-Ane CH, Berthet JP, Alric P, Pegis JD, Rouviere P, Mary H. Management of descending necrotizing mediastinitis: an aggressive treatment for an aggressive disease. Ann Thorac Surg. 1999;68(1):212-217.

7. Bayarri Lara CI, Sevilla Lopez S, Sanchez-Palencia Ramos A, Alkourdi Martinez A, Hernandez Escobar F, Quero Valenzuela F, Ruiz Zafra FJ, et al. [Surgical management of descending necrotizing mediastinitis]. Cir Esp. 2013;91(9):579-583.

8. Akman C, Kantarci F, Cetinkaya S. Imaging in mediasti- 
nitis: a systematic review based on aetiology. Clin Radiol. 2004;59(7):573-585.

9. Misawa Y, Fuse K, Hasegawa T. Infectious mediastinitis after cardiac operations: computed tomographic findings. Ann Thorac Surg. 1998;65(3):622-624.

10. Miller WD, Furst IM, Sandor GK, Keller MA. A prospective, blinded comparison of clinical examination and computed tomography in deep neck infections. Laryngoscope. 1999;109(11):1873-1879.

11. Uwa N, Terada T, Saeki N, Sagawa K, Ogino K, Sakagami M. Clinical features of 5 patients with descending necrotizing mediastinitis originating from deep neck in- fection. Auris Nasus Larynx. 2010;37(4):530-534.

12. Baskett RJ, MacDougall CE, Ross DB. Is mediastinitis a preventable complication? A 10-year review. Ann Thorac Surg. 1999;67(2):462-465.

13. Sposato LA, Yap CH, Powar N, Alizzi AM, Tatoulis J, Newcomb AE. Acute mediastinitis secondary to venous perforation by a peripherally inserted central venous catheter. J Cardiothorac Vasc Anesth. 2009;23(5):672-674.

14. Eroglu A, Kurkcuoglu C, Karaoglanoglu N, Tekinbas C, Cesur M. Spontaneous esophageal rupture following severe vomiting in pregnancy. Dis Esophagus. 2002;15(3):242-243. 